\title{
Comparison of the predictive values of CRP, CRP/albumin, RDW, neutrophil/lymphocyte, and platelet/lymphocyte levels in determining the severity of acute pancreatitis in patients with acute pancreatitis according to the BISAP score
}

\author{
Karabuga $\mathrm{B}^{1}$, Gemcioglu $\mathrm{E}^{2}$, Konca Karabuga $\mathrm{E}^{2}$, Baser $\mathrm{S}^{1}$, Ersoy $\mathrm{O}^{3}$ \\ Ankara City Hospital, Department of Internal Medicine, Ankara, Turkey. egemcioglu@gmail.com
}

\begin{abstract}
BACKGROUND: In our study, it was aimed to find out whether it would be possible to determine the disease severity by comparing the values of red cell distribution width (RDW), c-reactive protein (CRP), CRP/albumin, neutrophil/lymphocyte ratio (NLR), and platelet/lymphocyte ratio (PLR) in patients with acute pancreatitis according to Bedside Index of Severity in Acute Pancreatitis (BISAP) scores at the time of admission. METHODS: Five hundred patients diagnosed with acute pancreatitis were included in the study. RESULTS: According to BISAP scores, $388(77.6 \%)$ patients were evaluated as having mild acute pancreatitis and $112(22.4 \%)$ patients as having severe acute pancreatitis. In ROC analysis, values of $70.54 \%$ sensitivity and $70.10 \%$ specificity for CRP, $71.43 \%$ sensitivity and $70.88 \%$ specificity for CRP/albumin, $80.36 \%$ sensitivity and $30.30 \%$ specificity for RDW, $75.00 \%$ sensitivity and $43.98 \%$ specificity for NLR, and $55.36 \%$ sensitivity and $38.51 \%$ specificity for PLR were determined. Values of $85.71 \%$ sensitivity and $66.49 \%$ specificity were determined for the NLR-CRP/Albumin-RDW $2^{\star *}$ score as a newly created scoring system. CONCLUSION: CRP/albumin, NLR, PLR, and RDW values were found to be statistically significantly higher in patients with severe acute pancreatitis compared to those with mild acute pancreatitis according to the BISAP score $(p<0.001)$. In comparison to previous studies in the literature, similar sensitivity and specificity values were obtained in determining the severity of the disease with the new NLR-CRP/Albumin-RDW $2^{* *}$ score, and it is thought that this scoring system could be a practical and reliable guide in the treatment and follow-up of patients with acute pancreatitis (Tab. 6, Fig. 3, Ref. 22). Text in PDF www.elis.sk KEY WORDS: bedside index of severity in acute pancreatitis, BISAP, pancreatitis score, disease severity, scoring systems.
\end{abstract}

\section{Introduction}

Acute pancreatitis is a disease characterized by inflammation in the pancreatic parenchyma, with many different factors in its etiology. Many different causes are blamed in the etiology, especially gallstones and alcohol. Many scoring systems and parameters are used to determine the severity of acute pancreatitis and the Bedside Index of Severity in Acute Pancreatitis (BISAP) score is currently one of the most widely used scoring systems. The BISAP score has been compared with many scoring systems used to determine the severity of this disease. In one such study,

${ }^{1}$ Yildirim Beyazit University School of Medicine, Department of Internal Medicine, Ankara, Turkey, ${ }^{2}$ Ankara City Hospital, Department of Internal Medicine, Ankara, Turkey, and ${ }^{3}$ Yildirim Beyazit University School of Medicine, Department of Gastroenterology, Ankara, Turkey

Address for correspondence: E. Gemcioglu, MD, Ankara City Hospital, Department of Internal Medicine, Ankara 06100, Turkey.

Phone: +90.505.9369141, Fax: +90.312.3100194
BISAP and Ranson scores were compared to determine the severity of acute pancreatitis and the sensitivities of the Ranson and BISAP scores were found to be $97.4 \%$ and $69.2 \%$, respectively, while their specificity values were found to be similar (1). In another study, the sensitivity of the BISAP score in determining the severity of acute pancreatitis was $56 \%$ and its specificity was $91 \%(2)$.

Many different inflammatory markers such as neutrophil/lymphocyte ratio (NLR), platelet/lymphocyte ratio (PLR), c-reactive protein (CRP), CRP/albumin ratio, and red cell distribution width (RDW) levels are also used to determine the severity of acute pancreatitis. In a study by Wang et al. which included 110 patients diagnosed with acute pancreatitis. NLR, PLR, and RDW levels were compared and NLR was found to have the highest capacity to indicate the severity of acute pancreatitis due to hypertriglyceridemia (3). In a study by Liu et al. which included 279 patients, RDW level was found to have a prognostic value similar to that of the BISAP score in demonstrating the development of persistent organ failure in cases of acute pancreatitis (4). In a study by 
129-135

Kaplan et al. which included 192 patients with acute pancreatitis, the Ranson and Atlanta criteria were compared with the CRP/ /albumin ratio, while the latter was found to be an independent indicator for determining overall survival in patients with acute pancreatitis (5).

Although there are many studies in the literature in which CRP, CRP/albumin, NLR, PLR, and RDW values are examined separately for many diseases, including acute pancreatitis, no comprehensive study has been conducted with a large population to examine all of those parameters together and compare them with the BISAP scoring system, which has been shown to be the most valuable scoring system in determining the severity of acute pancreatitis in the first 12 hours of symptoms.

In this study, we aimed to determine whether those parameters can be used in determining the severity of acute pancreatitis by comparing the RDW, CRP, CRP/albumin, NLR, and PLR values in patients diagnosed with acute pancreatitis according to their BISAP scores at the time of admission.

\section{Materials and methods}

Five hundred patients who were hospitalized in Ankara City Hospital's internal medicine and gastroenterology clinics between February 2019 and March 2020 were included in our study. The values of platelets, lymphocytes, neutrophils, RDW, CRP, and albumin from routine blood counts of the patients at the time of admission to the hospital were reviewed retrospectively. Blood urea nitrogen (BUN) levels, age of the patient, Glasgow Coma Scale score obtained from physical examination at the time of admission, and the information on whether the patient met the systemic inflammatory response syndrome (SIRS) criteria, as well as whether there was pleural effusion obtained from posteroanterior chest X-ray or thorax computed tomography examinations were noted.

Diagnostic criteria of acute pancreatitis were considered as follows:

- Acute onset of girdle-style abdominal pain hitting the back

- Increase in serum amylase and lipase values more than 3 times normal

- Heterogeneity in the pancreatic parenchyma in radiological imaging, contamination in the fatty tissue around the parenchyma

The diagnosis was made with the presence of at least two of the findings (6).

The BISAP score is based on the following findings:

- Prescence of pleural effusion

- Meeting at least two of the SIRS criteria

- Age $>60$ years

- Glasgow Coma Scale score of $<15$

- Serum BUN level of $>25 \mathrm{mg} / \mathrm{dL}$

Those with a score of 3 or above while receiving 1 point for each positive criterion were defined as having severe acute pancreatitis, and those with a score of 2 or less were defined as having mild acute pancreatitis according to the BISAP scoring system (7).
Three different new scoring systems were created using NLR, CRP/albumin ratio, and RDW parameters and threshold values were determined for each of them.

Accordingly, in the scoring system where NLR and CRP/ /albumin values were used, the following threshold values were considered:

- NLR $>9$

- CRP/albumin $>0.0015$

In this evaluation, each criterion was evaluated as 1 point or more being positive and 0 points as being negative.

In the scoring systems where NLR, CRP/albumin, and RDW parameters were used, the following threshold values were considered.

$-\mathrm{NLR}>9$

- CRP/albumin $>0.0015$

- RDW $>13.65$

In the second evaluation, cases with 1 or more points were evaluated as positive and those with 0 points were evaluated as negative while assigning 1 point for each criterion that was met.

In the third evaluation, cases with 2 or more points were evaluated as positive and those with points in range of $0-1$ were evaluated as negative while assigning 1 point for each criterion that was met.

Inclusion criteria for the study were as follows:

- Being 18 years or older

- Having been diagnosed with acute pancreatitis

Exclusion criteria were as follows:

- Being diagnosed with active malignancy

- Pregnancy

- Missing or inaccessible data

The permission was obtained from the Ankara Yildirim Beyazit University Clinical Research Ethics Committee for this study.

\section{Data analysis and statistics}

Data analysis and statistical evaluation were performed with the help of SPSS 17.0. The conformity of the variables to normal distribution was examined by histogram graphics and the Kolmogorov-Smirnov test. Mean, standard deviation, and median values were used in the presentation of descriptive analyses. Categorical variables were compared with the Pearson chi-

Tab. 1. Demographic characteristics of the patients.

\begin{tabular}{lcc}
\hline & $\mathrm{n}$ & $\%$ \\
\hline Male & 247 & 49.40 \\
Female & 253 & 50.60 \\
BISAP score of $<3$, mild acute pancreatitis & 388 & 77.60 \\
BISAP score of $\geq 3$, severe acute pancreatitis & 112 & 22.40 \\
Nonbiliary acute pancreatitis & 139 & 27.80 \\
Biliary acute pancreatitis & 361 & 72.20 \\
Diabetes mellitus & 103 & 20.60 \\
Hypertension & 183 & 36.60 \\
Coronary artery disease & 74 & 14.80 \\
Other & 151 & 30.20 \\
Mortality & 23 & 4.60 \\
\hline
\end{tabular}

BISAP - Bedside Index of Severity in Acute Pancreatitis 
square test. The Mann-Whitney U test was used when evaluating nonnormally distributed (nonparametric) variables between two groups. The ability of CRP, CRP/albumin, RDW, NLR, and PLR values to predict severe acute pancreatitis was investigated by ROC analysis. Values of $\mathrm{p}<0.05$ were accepted as significant in statistical analysis.

Tab. 2. Laboratory parameters of the patients.

\begin{tabular}{lccccc}
\hline & Mean & SD & Median & P 25 & P 75 \\
\hline WBC & 11.90 & \pm 4.74 & 11.41 & 8.41 & 14.59 \\
Platelets & 263.59 & \pm 87.26 & 252.00 & 205.50 & 304.00 \\
Lymphocytes & 1.92 & \pm 10.72 & 1.29 & 0.85 & 1.78 \\
Neutrophils & 9.65 & \pm 4.67 & 9.10 & 5.97 & 12.39 \\
NLR & 10.21 & \pm 10.31 & 6.91 & 3.76 & 12.92 \\
PLR & 252.63 & \pm 194.83 & 192.52 & 136.15 & 302.37 \\
RDW & 14.18 & \pm 1.32 & 13.90 & 13.30 & 14.90 \\
CRP & 0.0661 & \pm 0.0790 & 0.0320 & 0.0090 & 0.0940 \\
Albumin & 40.15 & \pm 5.15 & 40.00 & 37.00 & 44.00 \\
CRP/albumin & 0.00181 & \pm 0.00232 & 0.00083 & 0.00021 & 0.00250 \\
\hline WBC - white blood cell count; NLR - neutrophil/lymphocyte ratio; PLR - plate- \\
let/lymphocyte ratio; RDW - red cell distribution width; CRP - c-reactive protein
\end{tabular}

\section{Results}

Of the patients included in our study, 247 (49.4\%) were male, $253(50.6 \%)$ were female, and $215(43 \%)$ patients were 60 years or older, which is the age threshold in BISAP scoring. According to BISAP scoring, $388(77.6 \%)$ patients were evaluated as having mild acute pancreatitis and $112(22.4 \%)$ patients as having severe acute pancreatitis. Other demographic characteristics of the patients are given in Table 1.

The mean age of the patients was $55.68 \pm 18.30$ years. Other laboratory parameters of the patients are given in Table 2 .

In our study, the percentage of patients aged 60 or over in the severe acute pancreatitis group was found to be statistically significantly higher according to BISAP scoring ( $\mathrm{p}<0.001$ ). Although the number of female patients in the severe acute pancreatitis group was higher than in the mild acute pancreatitis group, no statistically significant difference in terms of gender was found $(p=0.617)$. Demographic characteristics of the mild and severe acute pancreatitis groups are given in Table 3 .

In our study, age and white blood cell (WBC), neutrophil, NLR, PLR, RDW, $\mathrm{CRP}$, and CRP/albumin values in the patients from the severe acute pancreatitis group were found to be statistically significantly higher than in those from the mild acute pancreatitis group. Platelet, lymphocyte, and albumin levels were statistically significantly lower in the severe acute pancreatitis group $(\mathrm{p}<0.001)$. The comparison of the laboratory parameters of the mild and severe acute pancreatitis groups is given in Table 4.

The capacity of CRP, CRP/albumin, RDW, NLR, and PLR values to predict severe acute pancreatitis was investigated by ROC analysis and cut-off values were determined. When the value of 0.0545 was taken as the cut-off value for CRP, $70.54 \%$ sensitivity, $70.10 \%$ specificity, $40.51 \%$ positive predictive value (PPV), and $89.18 \%$ negative predictive value (NPV) were obtained. When the value of 0.00150 was taken as the cut-off value for CRP/albumin, the sensitivity of $71.43 \%$,

$\begin{array}{llllllll}\text { CRP/Albumin } & 0.00132 & \pm 0.00179 & 0.00053 & 0.00351^{*} & \pm 0.00303 & 0.00263 & <0.00\end{array}$

Mann-Whitney U test; * - Statistically significant, WBC - white blood cell count; NLR - neutrophil/lymphocyte ratio; PLR - platelet/lymphocyte ratio; RDW - red cell distribution width; CRP - c-reactive protein

Tab. 5. Specificity and sensitivity values of the measured parameters in determining the severity of acute pancreatitis.

\begin{tabular}{|c|c|c|c|c|c|c|c|c|c|}
\hline & \multirow{2}{*}{ Area } & \multirow{2}{*}{ SD Error } & \multirow{2}{*}{$\mathrm{p}$} & \multicolumn{2}{|c|}{$95 \%$ Confidence interval } & \multirow{2}{*}{ Sensitivity } & \multirow{2}{*}{ Specificity } & \multirow{2}{*}{ PPV } & \multirow{2}{*}{ NPV } \\
\hline & & & & Lower limit & Upper limit & & & & \\
\hline $\mathrm{CRP}>0.0545$ & 0.720 & 0.029 & $<0.001$ & 0.673 & 0.785 & $70.54 \%$ & $70.10 \%$ & $40.51 \%$ & $89.18 \%$ \\
\hline CRP/albumin $>0.0015$ & 0.740 & 0.029 & $<0.001$ & 0.684 & 0.796 & $71.43 \%$ & $70.88 \%$ & $41.45 \%$ & $89.58 \%$ \\
\hline RDW > $>13.65$ & 0.679 & 0.028 & $<0.001$ & 0.625 & 0.734 & $80.36 \%$ & $30.30 \%$ & $46.65 \%$ & $89.16 \%$ \\
\hline NLR $>9$ & 0.779 & 0.025 & $<0.001$ & 0.730 & 0.828 & $75.00 \%$ & $43.98 \%$ & $72.42 \%$ & $90.94 \%$ \\
\hline PLR $>263.50$ & 0.682 & 0.029 & $<0.001$ & 0.626 & 0.738 & $55.36 \%$ & $38.51 \%$ & $74.48 \%$ & $85.25 \%$ \\
\hline
\end{tabular}

ROC analysis, NLR - neutrophil/lymphocyte ratio; PLR - platelet/lymphocyte ratio; RDW - red cell distribution width; CRP - c-reactive protein; PPV - positive predictive value; NPV - negative predictive value 

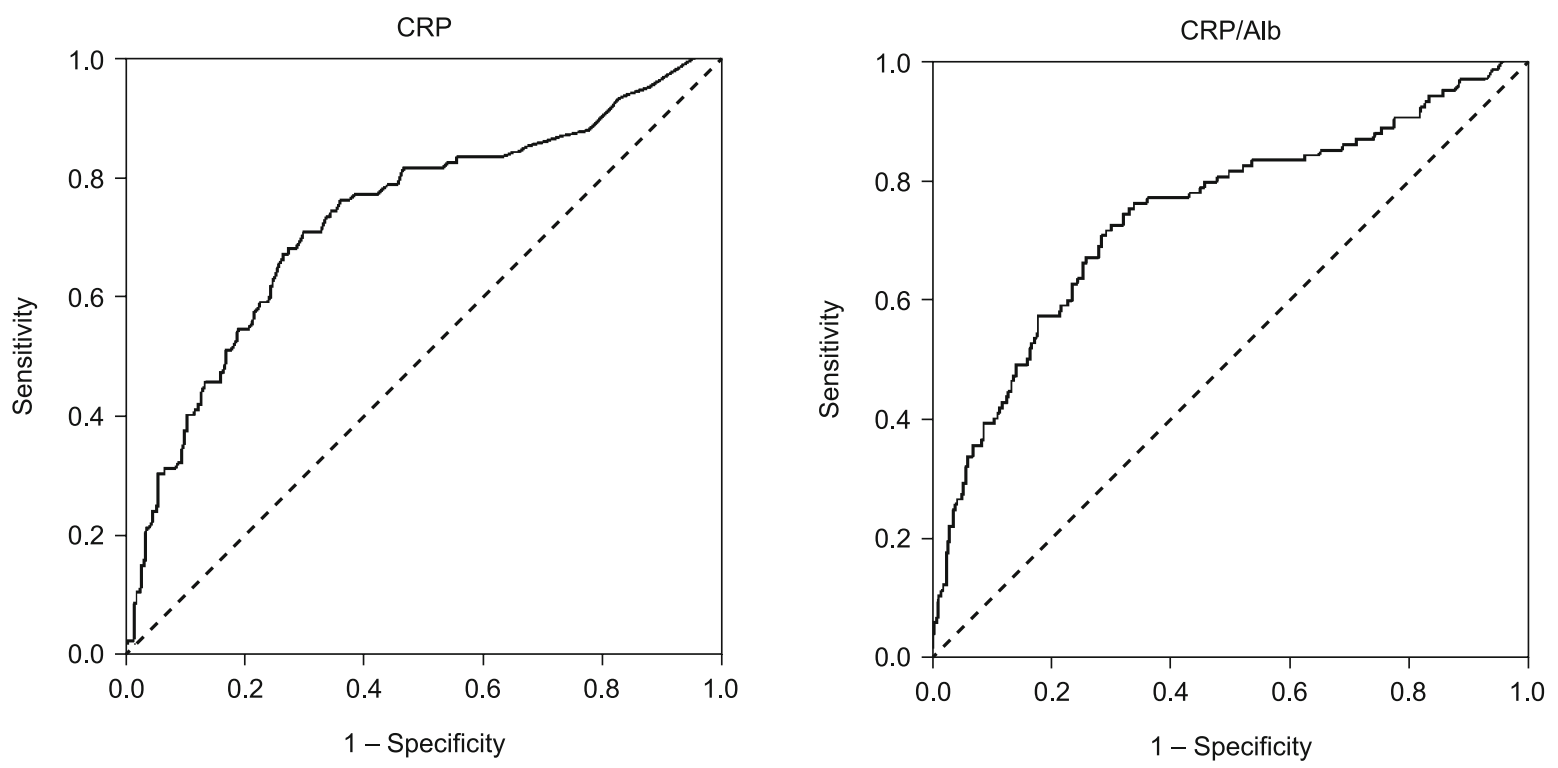

Fig. 1. Specificity and sensitivity of C-reactive protein (CRP) and CRP/albumin (CRP/Alb) values in determining the severity of acute pancreatitis.
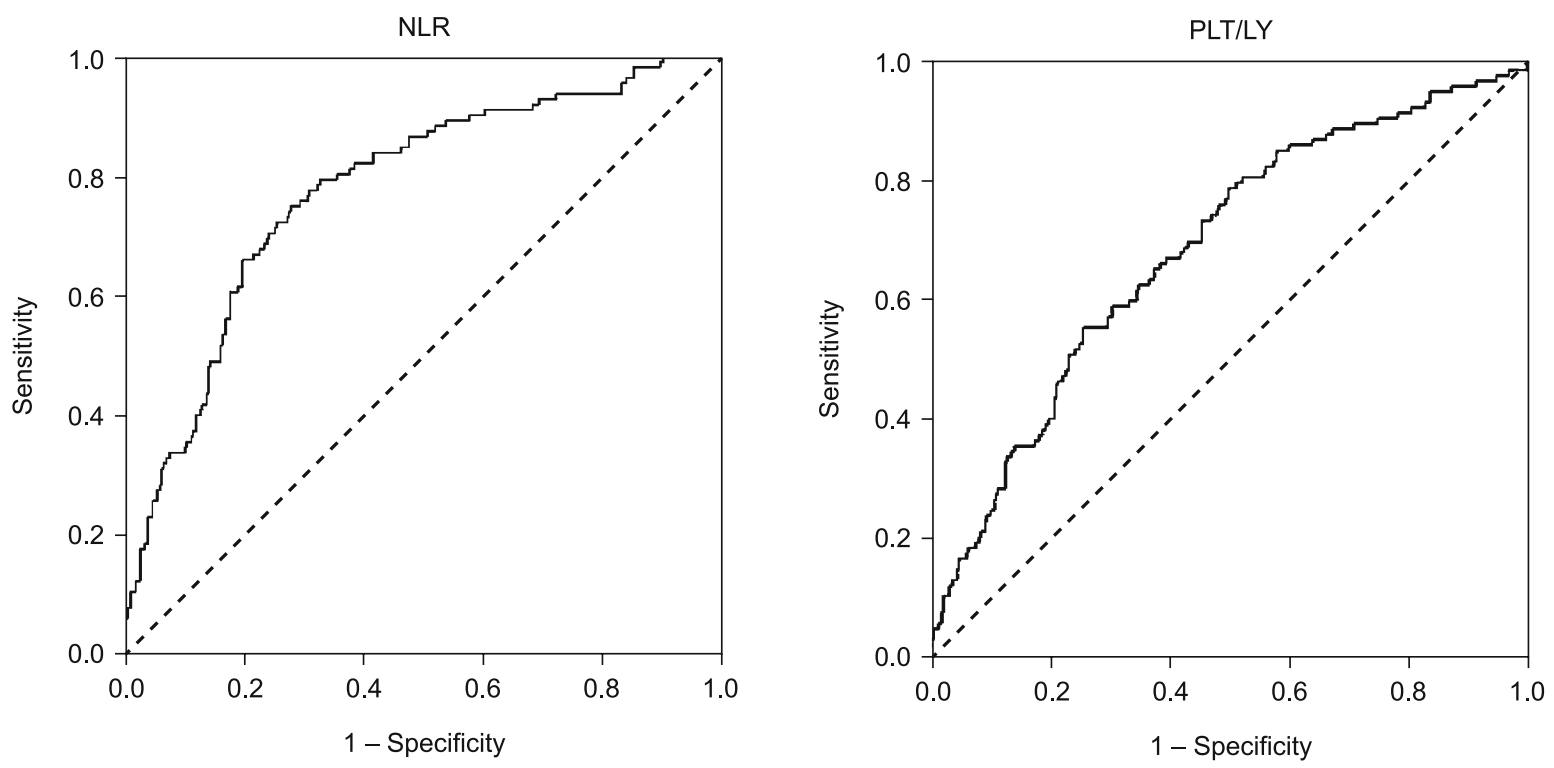

Fig. 2. Specificity and sensitivity of neutrophil/lymphocyte ratio (NLR) and platelet/lymphocyte ratio (PLT/LY) values in determining the severity of acute pancreatitis.

specificity of $70.88 \%$, PPV of $41.45 \%$, and NPV of $89.58 \%$ were obtained. When the cut-off value of 13.65 was taken for RDW, $80.36 \%$ sensitivity, $30.30 \%$ specificity, $46.65 \% \mathrm{PPV}$, and $89.16 \% \mathrm{NPV}$ were obtained. When the value of 9 was taken as the cut-off value for NLR, $75.00 \%$ sensitivity, $43.98 \%$ specificity, $72.42 \% \mathrm{PPV}$, and $90.94 \% \mathrm{NPV}$ were obtained. When the value of 263.50 was taken as the cut-off value for PLR, $55.36 \%$ sensitivity, $38.51 \%$ specificity, $74.48 \% \mathrm{PPV}$, and $85.25 \% \mathrm{NPV}$ were obtained. The specificity and sensitivity values are given in Table 5 (Figs 1, 2, 3).

The sensitivity, specificity, PPV, and NPV values found for NLR and CRP/albumin, which were determined as the most reliable parameters according to ROC analysis, and RDW, which was the parameter with the highest sensitivity value, are given in Table 6. 
Tab. 6. Specificity and sensitivity values of scoring systems created according to NLR-CRP/ /Albumin and NLR-CRP/Albumin-RDW threshold values.

\begin{tabular}{lcccc}
\hline & Sensitivity, \% & Specificity, $\%$ & PPV, \% & NPV, \% \\
\hline NLR-CRP/Albumin* & 89.29 & 44.07 & 31.55 & 93.44 \\
NLR-CRP/Albumin-RDW 1** & 99.11 & 19.07 & 26.12 & 98.67 \\
NLR-CRP/Albumin-RDW 2** & 85.71 & 66.49 & 42.48 & 94.16 \\
\hline
\end{tabular}

*: 1 point for each parameter, total minimum of 0 and maximum of 2 points; 1 point or more, positive; 0 points, negative, **: 1 point for each parameter, total minimum of 0 and maximum of 3 points; 1 point or more, positive; $0-1$ points, negative, $* * *: 1$ point for each parameter, total minimum of 0 and maximum of 3 points; $2-3$ points, positive; $0-1$ points, negative

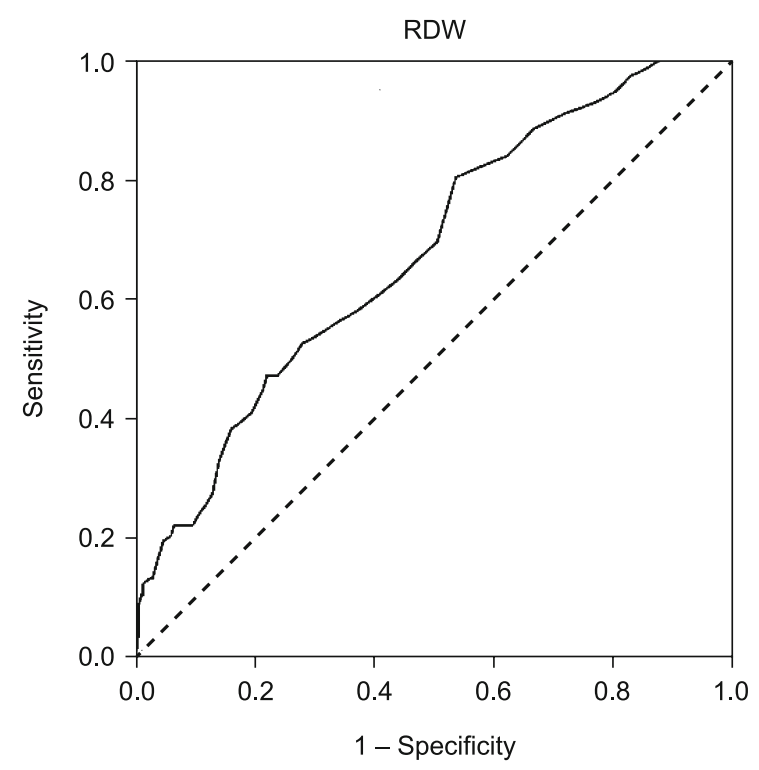

Fig. 3. Specificity and sensitivity of red cell distribution width (RDW) value in determining the severity of acute pancreatitis.

\section{Discussion}

In our study, CRP, CRP/albumin, RDW, NLR, and PLR values were compared according to BISAP scores in patients hospitalized with the diagnosis of acute pancreatitis in internal medicine and gastroenterology clinics, and their values in predicting the severity of acute pancreatitis were determined. NLR, PLR, RDW, CRP, and $\mathrm{CRP} /$ albumin values were statistically significantly higher in the severe acute pancreatitis group than in the mild acute pancreatitis group according to BISAP scores.

It is known that the severity of acute pancreatitis increases with age, and although the overall mortality is $1 \%$, mortality also increases with age (8). In our study, the mean age of the patients in the severe acute pancreatitis group was found to be statistically significantly higher than that in the mild acute pancreatitis group. In a study by Mandalia et al, it was stated that mortality rates increased at the age of 70 and above (9). A gender-based generalization cannot be made for the frequency of acute pancreatitis. Because of the high incidence of gallstones in female patients, acute biliary pancreatitis is more common in women, while alcohol-related pancreatitis is more common in men (10). In our study, the number of female patients was found to be higher than that of male patients, which is consistent with the literature. In parallel with the literature, the mortality rate among our severe acute pancreatitis patients according to BISAP scoring was significantly higher than that among mild acute pancreatitis patients. It has been reported in many studies that the parameters used in the BISAP scoring, such as high BUN, presence of pleural effusion, age of 60 or over, and presence of SIRS criteria increase the mortality rate of acute pancreatitis (11, 12). In a study by Arif et al, the Ranson and BISAP scores were compared for detecting severe acute pancreatitis with an accuracy rate of $82.0 \%$ for the Ranson score and $76.2 \%$ for the BISAP score (1). This information from the literature, which is similar to the findings of our study, illustrates the value of BISAP scoring in showing the severity of the disease. In a study by Kong et al, the predictive value of NLR in determining the severity of disease in patients with acute pancreatitis was examined, and when NLR was compared with the APACHE II and Ranson scores, it was found to have moderate sensitivity and specificity with a similar diagnostic value (13). In our study, the most reliable parameter in showing the severity of acute pancreatitis was the NLR value. NLR indicates the balance of neutrophils, which act as inflammatory activators, and lymphocytes, which act as regulators. Therefore, the higher the NLR, the higher the degree of inflammation is (14). NLR level is considered a reliable marker in determining the prognosis of disease in events involving inflammatory processes and various infective processes (15). Our results show similarity with those found in the literature.

Although platelet migration and destruction in the area of inflammation, susceptibility to prothrombotic events, and decrease in platelet levels may occur, the PLR may still give more accurate results since a lymphocyte decrease will also occur. The PLR has also been examined in acute pancreatitis patients. In a study including 243 patients, PLR was found to be significantly higher in biliary acute pancreatitis patients, with the predictive value of PLR reported to be higher than that of CRP (16). In our study, the CRP value was seen to be a more reliable parameter than that of PLR. This may be due to the larger population in our study. In the study conducted by Liu et al, $95.84 \%$ sensitivity and 44.71 $\%$ specificity were determined for PLR in detecting organ failure in acute pancreatitis patients, while the sensitivity of the BISAP score was $70.83 \%$ (4).

In our study, the CRP/albumin ratio was found to be the second most reliable parameter to NLR in determining the severity of the disease. CRP is a proinflammatory positive acute phase reactant. In malignant diseases and infective and inflammatory processes, the CRP value increases and the value of albumin, which is a negative acute phase reactant, decreases, and so the CRP/ /albumin ratio can provide more meaningful information about the inflammatory process. In a study by Yilmaz et al in which the value of the CRP/albumin ratio in determining the prognosis of disease according to the Ranson score in acute pancreatitis patients was examined, the threshold value for CRP/albumin was 
determined as $8.51 \mathrm{mg} / \mathrm{L}$ and the specificity and sensitivity values were $66 \%$ and $90 \%$, respectively (8). The difference between the results of the study of Yilmaz et al and our results may be due to the different reference scoring systems and threshold values. In a study by Yighit et al, it was stated that when the CRP value was evaluated together with the BISAP score, it could increase the success of the BISAP score in determining the severity of acute pancreatitis (17).

In contrast, RDW is significant in determining the severity of the disease but stands out as the least reliable parameter among those investigated. RDW is an inexpensive and easily accessible parameter that shows erythrocyte volume distribution and is an important inflammatory marker for many conditions such as infective processes, kidney dysfunction, cardiovascular diseases, and various malignant diseases (18). In a study by Gülen et al which included 322 patients diagnosed with acute pancreatitis, it was reported that RDW is a useful early-stage marker in predicting prognosis in nontraumatic acute pancreatitis cases (19). In a study conducted by Zhang et al, the RDW value, APACHE II score, and SOFA score were compared in patients with acute pancreatitis and achieved results in line with our study. They determined their cutoff value for RDW as 13.55 and obtained sensitivity of $83.3 \%$ and specificity of $66.7 \%$. It was concluded that the RDW value is superior to those scores (20).

Scoring systems created with the use of various parameters for common diseases play an important role in determining mortality and morbidity. In a study including 185 patients, the sensitivity of APACHE-II, one of the scoring systems used to determine the severity of acute pancreatitis, was found to be $70.3 \%$, while the sensitivity of Ranson scoring was $84.2 \%$, and the sensitivity of BISAP scoring was $37.5 \%$ (21). In a study including 303 patients, the sensitivity of determining the severity of acute pancreatitis of the BISAP score was $71.0 \%$, while the sensitivity of Ranson scoring was $74.2 \%$, the sensitivity of APACHE-II scoring was $71 \%$, and the sensitivity of Balthazar scoring was $51.6 \%$ (22). In our study, the most reliable parameters in determining the severity of acute pancreatitis were NLR and CRP/albumin, while the parameter with the highest sensitivity was the RDW value. Among the scoring systems created with these parameters, the sensitivity value of NLR-CRP/albumin-RDW 2** scoring was $85.71 \%$, the specificity value was $66.49 \%$, and the NPV was $94.16 \%$. The reason for achieving a higher sensitivity for this scoring system which we created may be that the number of patients included in our study was quite high compared to studies in the literature. More studies are needed to better understand the value of the scoring system we created in showing the severity of the disease.

\section{Conclusion}

Among the parameters examined in our study, NLR and $\mathrm{CRP} /$ albumin values were found to be the most reliable in determining the severity of acute pancreatitis, and RDW was the parameter with the highest sensitivity value. Values of $85.71 \%$ sensitivity, $66.49 \%$ specificity, and $94.16 \%$ NPV were obtained in the determination of disease severity by the NLR-CRP/albumin-RDW $2^{* *}$ scoring system, which was created using these parameters. Compared to studies in the literature, quite high values were obtained. It is thought that this scoring system, while being based on easily accessible parameters, will be a practical and reliable guide in the treatment and follow-up of patients with acute pancreatitis.

The strengths of our study are that it has a larger population as compared to previous studies in the literature, the values of RDW, CRP, CRP/albumin, PLR, and NLR were all examined together, and a new scoring system was developed to determine the severity of the disease with these values. The limitations of our study are that it was single-centered and that patient information was obtained retrospectively from patient files.

To the best of our knowledge, our study is the first one to determine the severity of disease in acute pancreatitis patients by examining and comparing the BISAP score, CRP, CRP/albumin, PLR, RDW, and NLR values, while being conducted on a large patient population of 500 patients. As a result, a new scoring system was created with those parameters. Our study is important in drawing attention to the usability of these inexpensive, easily accessible parameters in determining the severity of this disease.

\section{References}

1. Arif A, Jaleel F, Rashid K. Accuracy of BISAP score in prediction of severe acute pancreatitis. Pak J Med Sci 2019; 35 (4): 1008-1012. DOI: 10.12669/pjms.35.4.1286.

2. Gao W, Yang HX, Ma CE. The Value of BISAP Score for Predicting Mortality and Severity in Acute Pancreatitis: A Systematic Review and Meta-Analysis. PLoS One 2015; 10 (6): e0130412. DOI: 10.1371/journal.pone.0130412.

3. Wang Y, Fuentes HE, Attar BM et al. Evaluation of the prognostic value of neutrophil to lymphocyte ratio in patients with hypertriglyceridemia-induced acute pancreatitis. Pancreatology 2017; 17 (6): 893-897. DOI: 10.1016/j.pan.2017.10.001.

4. Liu G, Tao J, Zhu Z et al. The early prognostic value of inflammatory markers in patients with acute pancreatitis. Clin Res Hepatol Gastroenterol 2019; 43 (3): 330-337. DOI: 10.1016/j.clinre.2018.11.002.

5. Kaplan M, Ates I, Akpinar MY et al. Predictive value of C-reactive protein/albumin ratio in acute pancreatitis. Hepatobiliary Pancreat Dis Int 2017; 16 (4): 424-430. DOI: 10.1016/S1499-3872 (17)60007-9.

6. Papachristou GI, Whitcomb DC. Predictors of severity and necrosis in acute pancreatitis. Gastroenterol Clin North Am 2004; 33 (4): 871-890. DOI: 10.1016/j.gtc.2004.07.004.

7. Hagjer S, Kumar N. Evaluation of the BISAP scoring system in prognostication of acute pancreatitis - A prospective observational study. Int J Surg 2018; 54 (Pt A): 76-81. DOI: 10.1016/j.ijsu.2018.04.026.

8. Yilmaz EM, Kandemir A. Significance of red blood cell distribution width and C-reactive protein/albumin levels in predicting prognosis of acute pancreatitis. Turk J Trauma Emerg Surg 2018; 24 (6): 528-531. DOI: $10.5505 /$ tjtes.2018.98583.

9. Mandalia A, Wamsteker EJ, DiMagno MJ. Recent advances in understanding and managing acute pancreatitis. F1000Res 2018; 7: F1000 Faculty Rev-959. DOI: 10.12688/f1000research.14244.2. 
10. Yadav D, Lowenfels AB. The epidemiology of pancreatitis and pancreatic cancer. Gastroenterology 2013; 144 (6): 1252-1261. DOI: 10.1053/j. gastro.2013.01.068.

11. Barbara M, Tsen A, Rosenkranz L. Acute Pancreatitis in Chronic Dialysis Patients. Pancreas 2018; 47 (8): 946-951. DOI: 10.1097/ MPA.0000000000001119.

12. Mofidi R, Duff MD, Wigmore SJ et al. Association between early systemic inflammatory response, severity of multiorgan dysfunction and death in acute pancreatitis. Br J Surg 2006; 93 (6): 738-744. DOI: 10.1002/bjs.5290.

13. Kong W, He Y, Bao H et al. Diagnostic Value of Neutrophil-Lymphocyte Ratio for Predicting the Severity of Acute Pancreatitis: A MetaAnalysis. Dis Markers 2020; 2020:9731854. DOI: 10.1155/2020/9731854.

14. Huang L, Chen C, Yang $L$ et al. Neutrophil-to-lymphocyte ratio can specifically predict the severity of hypertriglyceridemia-induced acute pancreatitis compared with white blood cell. J Clin Lab Anal 2019; 33 (4): e22839. DOI: $10.1002 /$ jcla.22839.

15. Zahorec R. Neutrophil-to-lymphocyte ratio. Sixteen-year-long history since publication of our article in Bratislava Medical Journal. Bratisl Med J 2017; 118 (6): 321-323. DOI: 10.4149/BLL_2017_062.

16. Cho SK, Jung S, Lee KJ, Kim JW. Neutrophil to lymphocyte ratio and platelet to lymphocyte ratio can predict the severity of gallstone pancreatitis. BMC Gastroenterol 2018; 18 (1): 18. DOI: 10.1186/s12876018-0748-4.
17. Yigit Y, Selçok K. Can C-reactive Protein Increase the Efficiency of the Bedside Index of Severity in Acute Pancreatitis Scoring System? Cureus 2019; 11 (3): e4205. DOI: 10.7759/cureus. 4205.

18. Goyal H, Lippi G, Gjymishka A et al. Prognostic significance of red blood cell distribution width in gastrointestinal disorders. World J Gastroenterol 2017; 23 (27): 4879-4891. DOI: 10.3748/wjg.v23.i27.4879.

19. Gülen B, Sonmez E, Yaylaci S et al. Effect of harmless acute pancreatitis score, red cell distribution width and neutrophil/lymphocyte ratio on the mortality of patients with nontraumatic acute pancreatitis at the emergency department. World J Emerg Med 2015; 6 (1): 29-33. DOI: $10.5847 /$ wjem.j.1920-8642.2015.01.005.

20. Zhang FX, Li ZL, Zhang ZD et al. Prognostic value of red blood cell distribution width for severe acute pancreatitis. World J Gastroenterol 2019; 25 (32): 4739-4748. DOI: 10.3748/wjg.v25.i32.4739.

21. Papachristou GI, Muddana V, Yadav D et al. Comparison of BISAP, Ranson's, APACHE-II, and CTSI scores in predicting organ failure, complications, and mortality in acute pancreatitis. Am J Gastroenterol 2010; 105 (2): 435-442. DOI: 10.1038/ajg.2009.622.

22. Park JY, Jeon TJ, Ha TH et al. Bedside index for severity in acute pancreatitis: comparison with other scoring systems in predicting severity and organ failure. Hepatobiliary Pancreat Dis Int 2013; 12 (6): 645-650. DOI: 10.1016/s1499-3872 (13)60101-0.

Received August 23, 2021. Accepted September 21, 2021. 